\title{
New optical velocities of nearby dwarf LSB galaxies
}

\author{
D. I. Makarov ${ }^{1,2}$, I. D. Karachentsev ${ }^{1}$, and A. N. Burenkov ${ }^{1,2}$ \\ 1 Special Astrophysical Observatory, Russian Academy of Sciences, N. Arkhyz, KChR, 369167, Russia \\ 2 Isaac Newton Institute of Chile, SAO Branch, Russia \\ Received 11 February 2003 / Accepted 16 April 2003

\begin{abstract}
We present radial velocities for nearby dwarf galaxy candidates found by Karachentseva \& Karachentsev (1998) on the POSS-II films. Out of 118 observed objects, 88 have been detected in the $\mathrm{H}_{\alpha}$ line. Their median radial velocity
\end{abstract} \\ is $1750 \mathrm{~km} \mathrm{~s}^{-1}$. A quarter of the galaxies belong to the Local Volume, having corrected radial velocities $V_{\mathrm{LG}}<500 \mathrm{~km} \mathrm{~s}^{-1}$. \\ Some of them are members of the nearby groups around Maffei/IC 342, M 81, and NGC 6946.
}

Key words. galaxies: dwarf

\section{Introduction}

Kraan-Korteweg \& Tammann (1979) defined the Local Volume (LV) as our extragalactic neighbourhood out to $\sim 7 \mathrm{Mpc}$, which includes galaxies with radial velocities $V_{\mathrm{LG}}<500 \mathrm{~km} \mathrm{~s}^{-1}$ corrected to the Local Group centroid. Apparently, the LV census becomes increasingly incomplete for smaller galaxies, especially those of low surface brightness (LSB). Over the last two decades many observational teams undertook efforts to update the LV sample. As a result, the original Kraan-Korteweg \& Tammann list of 179 galaxies in the LV is now doubled. A significant number of nearby dwarf galaxies have been revealed in 1998-2001 by Karachentseva \& Karachentsev after visual inspection of the POSS-II films. On the northern sky they found about 400 nearby galaxy candidates with angular diameters $a \gtrsim 0.5$, being mainly LSB. All these objects were observed then in the HI line with the 100-m Effelsberg radio telescope (Huchtmeier et al. 1997, 2000a,b, 2003). About $50 \%$ of the galaxies were detected. Due to their median radial velocity, $1200 \mathrm{~km} \mathrm{~s}^{-1}$, and the median $\mathrm{HI}$ line width, $60 \mathrm{~km} \mathrm{~s}^{-1}$, most of the objects seem to be nearby dwarf galaxies, like DDO irregular galaxies. More than 50 of them are situated within the Local Volume.

As known, some nearby galaxies with heliocentric radial velocities $V_{\mathrm{h}}$ inside $[-300,+300] \mathrm{km} \mathrm{s}^{-1}$ remain still undetected or marginally detected in the HI line due to the Galactic emission. Gas rich galaxies can be undetected in $\mathrm{HI}$ when their radial velocities lie outside the interval $[-470,+3970] \mathrm{km} \mathrm{s}^{-1}$ used in the Effelsberg HI survey. Some target galaxies may be confused with their close bright neighbours, if their separations do not exceed the half power beam width of the Effelsberg telescope $(9.3$ at $21 \mathrm{~cm})$. For these reasons, measurements of optical velocities for nearby galaxy candidates are desirable.

Send offprint requests to: D. Makarov, e-mail: dim@sao.ru

\section{Observations}

The northern sky objects from the KK-lists (Karachentseva \& Karachentsev 1998; Karachentseva et al. 1999, 2001) were observed with the 6-m telescope of the Special Astrophysical Observatory of the Russian Academy of Sciences with the prime focus CCD spectrograph UAGS in 2000-2002. A $1 \mathrm{k} \times 1 \mathrm{k}$ pixels CCD with a pixel size of 24 microns together with a 1302 grooves/mm grating provide a dispersion of $1.2 \AA /$ pixel and a spectral resolution of $4 \AA$. The slit length and width were $150^{\prime \prime}$ and $2^{\prime \prime}$, respectively. A typical exposure time was $600-900 \mathrm{~s}$ in the $\mathrm{H}_{\alpha}$ region. The image processing was carried out with the LONG context in the ESO-MIDAS reduction package. We obtained about 230 spectra for 118 objects.

\section{Results}

The basic parameters of the observed objects are given in Table 1. Its columns contain:

(1) galaxy number in the lists of Karachentseva \& Karachentsev (1998) (KK); Karachentseva et al. (1999) (KKR); and Karachentsev et al. (2001) (KKH);

(2) equatorial coordinates at the 1950.0 epoch;

(3) major and minor angular diameters of the galaxy in arcmin, measured on blue plates;

(4) morphological type in the usual designations: Ir - irregular, Im - irregular magellanic, Sm - spiral magellanic; Sph dwarf spheroidal, PN? - probable planetary nebula;

(5) mean surface brightness in a scale: $\mathrm{H}$ - high (22$\left.23^{\mathrm{m}} / \square^{\prime \prime}\right), \mathrm{L}-$ low $\left(\sim 24^{\mathrm{m}} / \square^{\prime \prime}\right), \mathrm{VL}$ - very low $\left(\sim 25^{\mathrm{m}} / \square^{\prime \prime}\right)$, and EL - extremely low $\left(\sim 26^{\mathrm{m}} / \square^{\prime \prime}\right)$;

(6) heliocentric optical velocity and its internal error in $\mathrm{km} \mathrm{s}^{-1}$, measured from our spectra;

(7) heliocentric HI velocity and its error in $\mathrm{km} \mathrm{s}^{-1}$, measured by Huchtmeier et al. (2000a,b, 2003) and Karachentsev et al. (2001); blank lines in (6) and (7) mean that the objects were observed but not detected in $\mathrm{H}_{\alpha}$ or $\mathrm{HI}$, respectively; 
Table 1. List of northern objects from the KK-lists observed with the 6-m telescope.

\begin{tabular}{|c|c|c|c|c|c|c|c|c|c|}
\hline Object & \multicolumn{2}{|c|}{ RA (1950) Dec } & $a b$ & Type & SB & $V_{\mathrm{h}}(\mathrm{opt})$ & $V_{\mathrm{h}}(21 \mathrm{~cm})$ & $W$ & Notes \\
\hline 1 & \multicolumn{2}{|c|}{2} & 3 & 4 & 5 & 6 & 7 & 8 & 9 \\
\hline KKH 3 & 004300.3 & -012238 & 1.00 .7 & $\mathrm{Ir}$ & $\mathrm{L}$ & $4071 \pm 6$ & & 80 & \\
\hline KKH 6 & 013144.0 & +515010 & 0.80 .6 & $\mathrm{Ir}$ & $\mathrm{L}$ & $23 \pm 5$ & $53 \pm 1$ & & $*$ \\
\hline KKH 8 & 020947.1 & +100556 & 1.30 .7 & $\mathrm{Ir}$ & $\mathrm{L}$ & $1762 \pm 4$ & $1740 \pm 4$ & 30 & \\
\hline КKH 9 & 020958.8 & +323449 & 0.80 .5 & Sph? & $\mathrm{VL}$ & & & & \\
\hline KKH 15 & 025306.7 & +365602 & 0.70 .4 & $\mathrm{Ir}$ & $\mathrm{L}$ & $5117 \pm 9$ & $-142 \pm 2$ & 110 & $*$ \\
\hline KK 24 & 025354.5 & +171525 & 0.60 .4 & $\mathrm{Ir} ?$ & $\mathrm{~L}$ & $7188 \pm 5$ & & & \\
\hline KKH 16 & 025611.2 & +434047 & 1.30 .4 & $\mathrm{Ir}$ & $\mathrm{L}$ & $2186 \pm 8$ & $2148 \pm 2$ & 95 & \\
\hline KKH 17 & 025842.1 & +370431 & 0.50 .5 & $\mathrm{Ir}$ & $\mathrm{L}$ & $3722 \pm 6$ & & 65 & \\
\hline KKH 18 & 030000.5 & +332956 & 0.70 .3 & $\mathrm{Ir}$ & $\mathrm{L}$ & $203 \pm 5$ & $216 \pm 1$ & & \\
\hline ККH 20 & 031954.1 & +760559 & 0.90 .7 & $\mathrm{Ir}$ & $\mathrm{L}$ & $2165 \pm 10$ & $2131 \pm 1$ & 105 & \\
\hline KK 32 & 033726.8 & +193530 & 0.80 .7 & $\mathrm{Ir}$ & $\mathrm{L}$ & & & & \\
\hline KKH 23 & 033936.0 & +382730 & 1.10 .8 & $\mathrm{Ir}$ & $\mathrm{L}$ & $5466 \pm 7$ & & 75 & \\
\hline KK 35 & 034023.7 & +674226 & 2.51 .7 & $\mathrm{Ir}$ & $\mathrm{VL}$ & $-66 \pm 3$ & $105 \pm 2$ & & $*$ \\
\hline Cam A & 041926.1 & +724127 & 3.72 .1 & $\mathrm{Ir}$ & VL & $-49 \pm 3$ & $-47 \pm 1$ & & \\
\hline KKH 26 & 043541.4 & +065939 & 2.40 .6 & Ir? & $\mathrm{L}$ & $55 \pm 4$ & $-138 \pm 3$ & & $*$ \\
\hline KKH 28 & 044107.0 & +025421 & 1.10 .9 & $\mathrm{Ir}$ & $\mathrm{L}$ & $3483 \pm 6$ & $3508 \pm 2$ & & \\
\hline ККН 29 & 045327.3 & +375220 & 1.20 .7 & $\mathrm{Ir}$ & $\mathrm{L}$ & $-39 \pm 3$ & & & $*$ \\
\hline KK 49 & 053900.7 & +063928 & 0.70 .5 & $\mathrm{Ir}$ & $\mathrm{H}$ & $446 \pm 5$ & $455 \pm 2$ & 62 & \\
\hline КKH 33 & 054939.1 & +280518 & 1.10 .8 & PN & $\mathrm{L}$ & $49 \pm 3$ & & & $*$ \\
\hline КKH 37 & 063857.3 & +801035 & 1.20 .8 & $\mathrm{Ir}$ & $\mathrm{L}$ & $11 \pm 7$ & $-148 \pm 2$ & & $*$ \\
\hline KK 60 & 072023.0 & +460610 & 1.10 .4 & $\mathrm{Ir}$ & $\mathrm{L}$ & $5965 \pm 3$ & & & \\
\hline KK 65 & 073940.2 & +164047 & 0.90 .5 & $\mathrm{Ir}$ & $\mathrm{H}$ & $288 \pm 4$ & $279 \pm 1$ & & \\
\hline KKH 42 & 075737.3 & +861649 & 0.80 .5 & $\mathrm{Ir}$ & $\mathrm{L}$ & $1841 \pm 3$ & $1841 \pm 2$ & 35 & \\
\hline KK 67 & 080034.9 & +151703 & 1.00 .5 & $\mathrm{Ir}$ & $\mathrm{L}$ & $1991 \pm 4$ & & & \\
\hline ККH 46 & 090558.5 & +052943 & 0.60 .5 & $\mathrm{Ir}$ & $\mathrm{L}$ & $599 \pm 3$ & $598 \pm 1$ & & \\
\hline ККН 51 & 092724.2 & +201242 & 0.70 .7 & $\mathrm{Ir}$ & $\mathrm{L}$ & & $561 \pm 1$ & & \\
\hline КKH 52 & 093452.9 & +274735 & 0.60 .3 & $\mathrm{Ir}$ & $\mathrm{L}$ & $1610 \pm 3$ & $1594 \pm 2$ & & \\
\hline ККН 53 & 093753.2 & +001615 & 0.80 .7 & $\mathrm{Ir}$ & $\mathrm{L}$ & & & & \\
\hline КKH 54 & 094206.4 & +322807 & 0.90 .9 & $\mathrm{Ir}$ & $\mathrm{L}$ & $546 \pm 4$ & $538 \pm 1$ & & \\
\hline KK 78 & 094723.6 & +314126 & 0.50 .3 & $\mathrm{Ir}$ & $\mathrm{H}$ & $539 \pm 4$ & $520 \pm 2$ & 40 & $*$ \\
\hline KK 80 & 095045.0 & +294057 & 1.10 .6 & $\mathrm{Ir}$ & $\mathrm{L}$ & $4405 \pm 10$ & & 55 & \\
\hline ККН 58 & 100423.3 & +391250 & 1.60 .6 & $\mathrm{Ir}$ & $\mathrm{H}$ & $601 \pm 5$ & $587 \pm 3$ & & \\
\hline KK 86 & 100522.0 & +304409 & 1.00 .6 & Ir? & $\mathrm{L}$ & $6273 \pm 5$ & & & \\
\hline КKH 60 & 101322.0 & +070315 & 0.80 .4 & $\mathrm{Ir}$ & $\mathrm{L}$ & $286 \pm 8:$ & & & $*$ \\
\hline DDO 82 & 102647.0 & +705233 & 2.81 .9 & $\operatorname{Im}$ & $\mathrm{H}$ & $56 \pm 3$ & & & $*$ \\
\hline ККH 62 & 104151.2 & +542809 & 0.60 .4 & $\mathrm{Ir}$ & $\mathrm{L}$ & $1001 \pm 3$ & $999 \pm 2$ & & \\
\hline KK 95 & 104603.6 & +64 5920 & 2.21 .7 & $\mathrm{Ir}$ & $\mathrm{VL}$ & & & & $*$ \\
\hline KKH 64 & 104857.0 & +034310 & 1.20 .9 & $\operatorname{Im}$ & $\mathrm{L}$ & $1034 \pm 11$ & $1070 \pm 1$ & & \\
\hline KDG 73 & 104928.2 & +694842 & 0.60 .5 & $\mathrm{Ir}$ & $\mathrm{L}$ & & $116 \pm 1$ & & $*$ \\
\hline KK 97 & 105535.3 & +202235 & 0.70 .5 & $\mathrm{Ir}$ & $\mathrm{L}$ & $4270 \pm 8$ & & & \\
\hline KK 100 & 111122.9 & +113610 & 1.20 .5 & $\mathrm{Ir}$ & VL & $2969 \pm 5$ & & 80 & \\
\hline KKH 70 & 113642.0 & +602655 & 0.90 .4 & $\mathrm{Ir}$ & $\mathrm{H}$ & $1297 \pm 7$ & $-151 \pm 1$ & & $*$ \\
\hline КKH 74 & 115238.6 & +442543 & 1.00 .8 & $\mathrm{Ir}$ & $\mathrm{L}$ & & $727 \pm 3$ & & \\
\hline KK 118 & 115842.9 & +540301 & 0.60 .2 & $\mathrm{Ir}$ & VL & & & & \\
\hline КKH 75 & 115922.0 & +625513 & 0.70 .4 & $\mathrm{Ir}$ & $\mathrm{L}$ & & $-153 \pm 1$ & & $*$ \\
\hline KK 121 & 120252.3 & +435913 & 1.10 .6 & $\mathrm{Ir}$ & $\mathrm{VL}$ & & & & \\
\hline ККH 76 & 120850.6 & +384906 & 0.70 .3 & $\mathrm{Ir}$ & $\mathrm{H}$ & $1078 \pm 4$ & $1081 \pm 2$ & & \\
\hline
\end{tabular}


Table 1. continued.

\begin{tabular}{|c|c|c|c|c|c|c|c|c|c|}
\hline Object & \multicolumn{2}{|c|}{ RA (1950) Dec } & $a b$ & Type & SB & $V_{\mathrm{h}}(\mathrm{opt})$ & $V_{\mathrm{h}}(21 \mathrm{~cm})$ & $W$ & Notes \\
\hline 1 & \multicolumn{2}{|c|}{2} & 3 & 4 & 5 & 6 & 7 & 8 & 9 \\
\hline KKH 77 & 121142.1 & +662212 & 1.50 .6 & $\mathrm{Ir}$ & $\mathrm{H}$ & $62 \pm 3$ & $68 \pm 2$ & & $*$ \\
\hline UGC 7298 & 121402.2 & +523029 & 1.10 .6 & $\mathrm{Ir}$ & $\mathrm{L}$ & & $173 \pm 1$ & & $*$ \\
\hline KK 130 & 121517.6 & +284509 & 0.60 .3 & $\mathrm{Ir}$ & $\mathrm{L}$ & & & & \\
\hline UGC 7369 & 121708.1 & +300939 & 1.01 .0 & E & $\mathrm{H}$ & & & & \\
\hline KKH 79 & 121728.0 & +614745 & 0.50 .3 & $\mathrm{Ir}$ & $\mathrm{L}$ & & $516 \pm 1$ & & \\
\hline KK 155 & 123513.0 & +072242 & 1.21 .0 & $\mathrm{Ir}$ & $\mathrm{L}$ & $64 \pm 5$ & & 80 & $*$ \\
\hline KKH 82 & 131043.1 & +420303 & 1.31 .0 & $\mathrm{Ir}$ & $\mathrm{L}$ & & & & \\
\hline KKH 83 & 132238.0 & +424509 & 0.90 .5 & Ir & $\mathrm{L}$ & $1096 \pm 5$ & $1105 \pm 2$ & 60 & \\
\hline KK 205 & 132646.8 & +675328 & 1.20 .5 & $\mathrm{Ir}$ & $\mathrm{L}$ & $991 \pm 5$ & & 60 & \\
\hline KK 206 & 133118.6 & +492130 & 1.00 .6 & $\mathrm{Ir}$ & $\mathrm{H}$ & $591 \pm 3$ & $588 \pm 2$ & 35 & \\
\hline КKH 84 & 133208.2 & +090256 & 1.10 .7 & $\mathrm{Ir}$ & $\mathrm{H}$ & $1215 \pm 3$ & $1233 \pm 3$ & & \\
\hline KK 209 & 133552.8 & +492226 & 0.40 .2 & $\mathrm{Ir}$ & $\mathrm{L}$ & & & & \\
\hline KKH 86 & 135202.2 & +042917 & 0.70 .5 & $\mathrm{Ir}$ & $\mathrm{L}$ & & $287 \pm 1$ & & \\
\hline KK 225 & 135251.5 & +375542 & 0.40 .3 & $\mathrm{Ir}$ & $\mathrm{L}$ & $5843 \pm 3$ & & & \\
\hline KKR 1 & 135516.8 & +080451 & 0.50 .3 & $\mathrm{Ir}$ & $\mathrm{L}$ & & & & \\
\hline KKH 87 & 141332.9 & +571910 & 0.80 .3 & $\mathrm{Ir}$ & $\mathrm{H}$ & $327 \pm 5$ & $320 \pm 1$ & & \\
\hline KKR 4 & 141357.0 & +140624 & 1.11 .1 & PN? & VL & $-17 \pm 2$ & & & $*$ \\
\hline KKR 7 & 141444.3 & +135459 & 0.60 .5 & $\mathrm{Ir}$ & $\mathrm{L}$ & $5013 \pm 6$ & & 75 & \\
\hline KK 231 & 141534.6 & +231821 & 0.80 .3 & Ir & $\mathrm{L}$ & $4635 \pm 4$ & & & \\
\hline KKR 8 & 141645.4 & +032108 & 1.30 .8 & Ir? & $\mathrm{L}$ & & & & \\
\hline KKR 10 & 143010.3 & +314345 & 0.60 .5 & Ir & $\mathrm{L}$ & $3415 \pm 4$ & & & \\
\hline KKR 14 & 145209.8 & +012150 & 0.50 .3 & $\mathrm{Ir}$ & $\mathrm{L}$ & $1830 \pm 7$ & & & \\
\hline KK 237 & 150646.1 & +562703 & 0.70 .5 & Ir? & $\mathrm{L}$ & & $-177 \pm 1$ & & \\
\hline KKR 17 & 150846.1 & +111313 & 0.40 .3 & $\mathrm{Ir}$ & $\mathrm{L}$ & $8273 \pm 4$ & & 40 & \\
\hline KKR 19 & 152044.7 & +572917 & 0.90 .6 & $\mathrm{Ir}$ & VL & $765 \pm 5$ & & & \\
\hline KKH 88 & 155051.0 & +641630 & 0.70 .5 & $\mathrm{Ir}$ & $\mathrm{H}$ & $923 \pm 3$ & $933 \pm 2$ & 45 & \\
\hline KKR 24 & 161125.5 & +023920 & 0.70 .5 & $\mathrm{Ir}$ & $\mathrm{L}$ & & & & \\
\hline KKR 25 & 161237.3 & +542946 & 1.10 .6 & Sph? & $\mathrm{L}$ & & $-139 \pm 1$ & & \\
\hline KKR 27 & 163847.8 & +220045 & 0.40 .2 & Ir? & $\mathrm{L}$ & $2819 \pm 6$ & & & \\
\hline KKR 28 & 164330.2 & +024439 & 1.00 .3 & Ir? & VL & & & & \\
\hline KKR 29 & 164613.8 & +222527 & 0.70 .3 & $\mathrm{Ir}$ & $\mathrm{L}$ & $2871 \pm 5$ & & & \\
\hline KKR 31 & 165627.4 & +231650 & 0.70 .3 & $\mathrm{Ir}$ & $\mathrm{L}$ & & & & \\
\hline KKR 32 & 165925.4 & +210755 & 0.40 .4 & $\mathrm{Ir}$ & $\mathrm{L}$ & $9436 \pm 5$ & & & \\
\hline KKR 35 & 172826.6 & +062225 & 0.40 .3 & $\mathrm{Ir}$ & $\mathrm{L}$ & & & & \\
\hline KKR 36 & 174345.0 & +020804 & 1.10 .8 & $\mathrm{Im}$ & $\mathrm{L}$ & $2988 \pm 6$ & & 40 & \\
\hline KKR 37 & 174457.1 & +222147 & 0.70 .4 & $\mathrm{Ir}$ & $\mathrm{L}$ & $6425 \pm 13$ & & 110 & \\
\hline KKR 38 & 174640.7 & +261301 & 0.80 .4 & $\mathrm{Ir}$ & $\mathrm{L}$ & $2836 \pm 13$ & & & \\
\hline KKR 41 & 180614.5 & +002210 & 1.81 .6 & PN? & EL & & & & \\
\hline KKR 43 & 181454.8 & +095804 & 0.70 .6 & $\mathrm{Ir}$ & $\mathrm{L}$ & $2189 \pm 5$ & & & \\
\hline KKR 44 & 183348.0 & +310218 & 0.70 .6 & Ir & $\mathrm{L}$ & $2931 \pm 4$ & & & \\
\hline KKR 46 & 193528.2 & +543133 & 0.80 .6 & Ir & $\mathrm{L}$ & $3734 \pm 8$ & $3779 \pm 5$ & & \\
\hline KKH 90 & 194150.0 & +682703 & 1.30 .7 & Ir? & VL & & $-139 \pm 1$ & & $*$ \\
\hline KKH 91 & 195530.6 & +043917 & 0.90 .5 & $\mathrm{Ir}$ & $\mathrm{L}$ & $-4 \pm 2$ & $-107 ?$ & & $*$ \\
\hline KKR 47 & 195554.0 & +420734 & 0.80 .4 & Ir & $\mathrm{L}$ & $4766 \pm 12$ & & & \\
\hline KKR 48 & 195714.7 & +622909 & 0.90 .4 & $\mathrm{Ir}$ & $\mathrm{L}$ & $3165 \pm 7$ & $3158 \pm 3$ & & $*$ \\
\hline KKR 50 & 200849.0 & +104642 & 0.60 .4 & $\mathrm{Ir}$ & $\mathrm{L}$ & $7541 \pm 8$ & & & \\
\hline KKH 92 & 200934.0 & +655603 & 0.50 .2 & $\mathrm{Ir}$ & $\mathrm{L}$ & $2650 \pm 11$ & & & \\
\hline KKR 51 & 201952.6 & +521826 & 0.70 .3 & Ir & $\mathrm{L}$ & $2963 \pm 5$ & & 40 & \\
\hline
\end{tabular}


Table 1. continued.

\begin{tabular}{|c|c|c|c|c|c|c|c|c|c|}
\hline Object & \multicolumn{2}{|c|}{ RA (1950) Dec } & $a b$ & Type & SB & $V_{\mathrm{h}}(\mathrm{opt})$ & $V_{\mathrm{h}}(21 \mathrm{~cm})$ & $W$ & Notes \\
\hline 1 & \multicolumn{2}{|c|}{2} & 3 & 4 & 5 & 6 & 7 & 8 & 9 \\
\hline KK 250 & 202914.4 & +601622 & 1.80 .8 & $\mathrm{Ir}$ & VL & $127 \pm 5$ & $127 \pm 2$ & & \\
\hline KK 251 & 202931.9 & +601103 & 1.60 .8 & $\mathrm{Ir}$ & VL & $147 \pm 6$ & $130 \pm 1$ & & \\
\hline KK 252 & 203033.5 & +603834 & 0.90 .9 & Ir & VL & $124 \pm 6$ & $138 \pm 1$ & & \\
\hline KKR 53 & 203049.1 & +012335 & 0.60 .3 & Ir? & $\mathrm{L}$ & $5873 \pm 8$ & & 120 & \\
\hline KKR 54 & 203323.7 & -012925 & 0.70 .3 & $\mathrm{Ir}$ & $\mathrm{L}$ & $1730 \pm 7$ & & & \\
\hline KK 254 & 203346.2 & +605512 & 1.50 .9 & Ir? & EL & & & & \\
\hline KKR 55 & 204415.2 & +601340 & 0.60 .4 & Ir & $\mathrm{L}$ & $23 \pm 5$ & $32 \pm 1$ & & $*$ \\
\hline KKR 56 & 204711.5 & +582556 & 0.70 .4 & Ir & $\mathrm{L}$ & $-43 \pm 8$ & $-135:$ & & $*$ \\
\hline KKR 57 & 204636.5 & +625303 & 0.50 .4 & Ir & $\mathrm{L}$ & $1553 \pm 4$ & & & \\
\hline KKR 58 & 204819.1 & +575504 & 2.10 .2 & $\mathrm{Sm}$ & VL & $2718 \pm 10$ & $2756 \pm 2$ & 170 & \\
\hline Ceph 1 & 204952.0 & +564206 & 3.01 .5 & $\mathrm{Sm} ?$ & VL & $33 \pm 7$ & $58 \pm 5$ & & $*$ \\
\hline ККН 93 & 205656.5 & +620915 & 0.50 .3 & $\mathrm{Ir}$ & $\mathrm{L}$ & $2539 \pm 11$ & $-112 \pm 2$ & & $*$ \\
\hline KKR 59 & 210202.8 & +570517 & 2.31 .4 & Ir & VL & $-3 \pm 4$ & 17: & & $*$ \\
\hline KKR 60 & 210430.6 & +570015 & 0.70 .5 & Ir & VL & $-14 \pm 7$ & & & $*$ \\
\hline KKR 62 & 212904.3 & +522824 & 1.10 .6 & Ir? & EL & $-27 \pm 6$ & & & $*$ \\
\hline KKR 63 & 215340.8 & +401405 & 0.60 .3 & $\mathrm{Ir}$ & $\mathrm{L}$ & $5679 \pm 12$ & & & \\
\hline KKR 64 & 215805.5 & +414715 & 1.00 .6 & Ir & VL & $4457 \pm 8$ & & & \\
\hline KKR 67 & 220412.3 & +370631 & 0.40 .3 & Ir? & $\mathrm{L}$ & $5798 \pm 7$ & & 70 & \\
\hline KKR 72 & 223348.4 & +232702 & 0.70 .5 & Ir? & $\mathrm{L}$ & & & & \\
\hline KKR 76 & 231909.1 & +254955 & 0.60 .4 & $\mathrm{Ir}$ & $\mathrm{L}$ & & & & \\
\hline KKR 78 & 232831.1 & +220957 & 0.50 .4 & Ir & $\mathrm{L}$ & $6904 \pm 6$ & & & \\
\hline КKH 98 & 234303.9 & +382624 & 1.10 .6 & Ir & $\mathrm{L}$ & $-134 \pm 4$ & $-137 \pm 1$ & & \\
\hline KKH100 & 234951.4 & +083345 & 0.60 .4 & Ir & $\mathrm{L}$ & $5530 \pm 6$ & & 80 & \\
\hline
\end{tabular}

Notes:

KKH 6. A probable member of the Maffei/IC 342 group.

KKH 15. This is UGC 2397, a distant peculiar galaxy. The HI emission is local.

KK 35. A peripheral star complex in IC 342, confusion with a strong HI from IC 342.

KKH 26. Not a galaxy, but a Galactic emission filament.

KKH 29. Probably not a galaxy, but a local HII region.

KKH 33. A planetary nebula PNG18.5+00.9.

KKH 37. Mailyan 16. The $21 \mathrm{~cm}$ velocity corresponds, apparently, to Galactic HII. A new Local Volume (LV) member? On its NE side there is a background galaxy with $V_{\mathrm{h}}=13220 \mathrm{~km} \mathrm{~s}^{-1}$.

KK 78. UGC 5272b (Hopp \& Schulte-Ladbeck 1991). The HI line confusion with UGC 5272 at 2'.

KKH 60. Should be confirmed as a probable new LV member. Two very faint emission details in two spectra.

DDO 82. A member of the M 81 group.

KK 95. UGCA $220=$ Mailyan 58, reflection nebula.

KDG 73. A member of the M 81 group. $V(\mathrm{HI})=-132 \pm 6 \mathrm{~km} \mathrm{~s}^{-1}(\mathrm{NED}), V(\mathrm{HI})=+116 \pm 1$ (Huchtmeier et al. 2003).

KKH 70. BCG, local HI.

KKH 75. Local HI?

KKH 77. UGC 7242, a peripheral member of the M 81 complex.

UGC 7298. A peripheral member of the M 81 complex, resolved with the HST.

KK 155. UGC $7795=$ DDO $139=$ VCC 1726, $V_{\mathrm{h}}(\mathrm{NED})=62 \pm 7 \mathrm{~km} \mathrm{~s}^{-1}$.

KKR 4. F650-01. A planetary nebula.

KKH 90. A reflection nebula.

KKH 91. An emission nebula.

KKR 48. There is also Galactic $\mathrm{H}_{\alpha}$.

KKR 55. Comet-like, resolved with the 6-m telescope, companion to NGC 6946.

KKR 56. Galactic HI, resolved with the 6-m telescope, companion to NGC 6946.

Ceph 1. Companion to NGC 6946, HI velocity from Barton et al. (1999).

KKH 93. Local HI.

KKR 59. The HI velocity distorted with the Galactic HI. The resolved companion to NGC 6946.

KKR 60. An emission nebula or a new companion to NGC 6946 ?

KKR 62. A planetary nebula. 

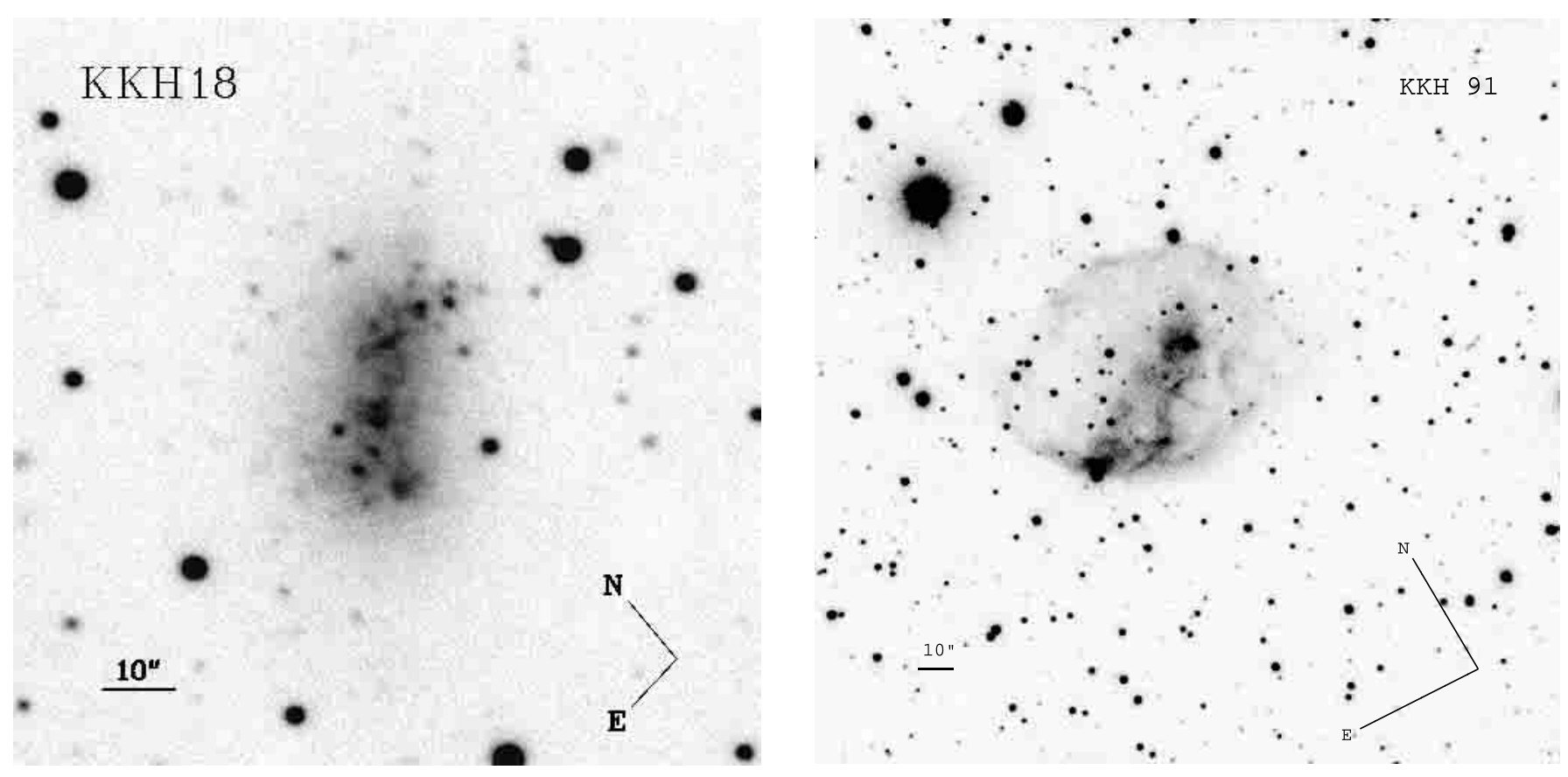

Fig. 1. CCD images of two LSB objects observed with the 6-m telescope: a) dwarf irregular galaxy KKH 18 imaged in the $V$ band, b) KKH 91, a new planetary nebula, imaged in $\mathrm{H}$-alpha filter by S. Kajsin.

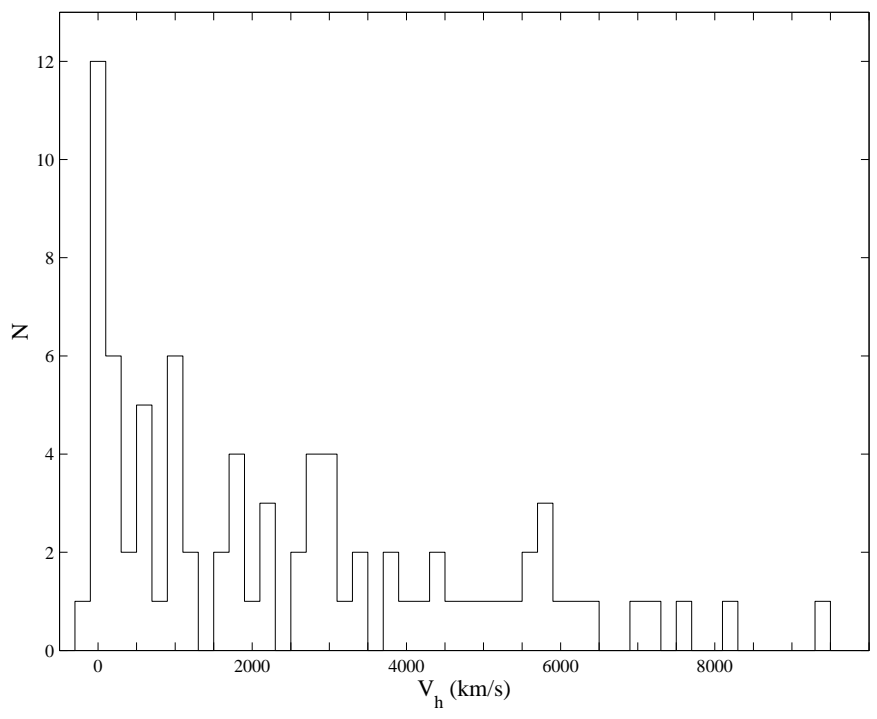

Fig. 2. Distribution of the observed galaxies according to heliocentric radial velocities in $\mathrm{km} \mathrm{s}^{-1}$.

(8) range of internal motions, $W$, in $\mathrm{km} \mathrm{s}^{-1}$ measured along the slit position. It corresponds usually to a difference between maximal and minimal measured velocity in cases significant internal motions;

(9) asterisk addressing the table footnotes.

As an illustration, we present in Fig. 1 large-scale CCD images of two objects obtained with the 6-m telescope: a semiresolved irregular galaxy, KKH 18, having $V_{\mathrm{LG}}=372 \mathrm{~km} \mathrm{~s}^{-1}$, and KKH 91, which turns out to be a planetary nebula.
In total we obtained spectra for 118 nearby galaxy candidates, and 88 of them were detected in the $\mathrm{H}_{\alpha}$ line. Figure 2 shows the object distribution according to their heliocentric radial velocity. The median velocity for the detected objects is $1750 \mathrm{~km} \mathrm{~s}^{-1}$.

When six local Galactic objects: KKH 26, KKH 29, KKH 33, KKR 4, KKH 91, and KKR 62 with heliocentric velocities in a range of $[-39,+55] \mathrm{km} \mathrm{s}^{-1}$ are excluded, the median velocity of the sample increases to $1920 \mathrm{~km} \mathrm{~s}^{-1}$. Therefore, the majority of the observed galaxies are true nearby dwarf galaxies situated inside the Local supercluster.

From 34 galaxies with optical as well radio velocities we obtained the mean-square velocity difference $\sigma\left(V_{\mathrm{opt}}-V_{\mathrm{HI}}\right)=$ $19 \mathrm{~km} \mathrm{~s}^{-1}$, and the mean velocity difference $\left\langle V_{\mathrm{opt}}-V_{\mathrm{HI}}\right\rangle=$ $-3 \pm 3 \mathrm{~km} \mathrm{~s}^{-1}$, showing a good agreement between $\mathrm{H}_{\alpha}$ and HI velocity measurements.

The derived optical spectra allow us to clarify the nature of some objects that have been marginally detected before (see Table footnotes). For instance, due to their velocities, KKH 6 , KK 35, and KK $41=$ Cam A are confirmed as members of the Maffei/IC 342 group. Reproductions of spectra of Cam A and KKH 6 are presented in Fig. 3, where a faint $\mathrm{H}$-alpha emission is seen in both the objects.

Further, the blue dwarf compact galaxy KK $78=$ UGC $5272 \mathrm{~b}$ is recognized by us as a companion to another dwarf galaxy, UGC 5272, with a radial velocity difference of only $(19 \pm 4) \mathrm{km} \mathrm{s}^{-1}$. Together with KKH 54, UGC 5209, and UGC 5186, they probably form a new nearby group of dwarf galaxies, as discussed by Tully et al. (2002).

A more accurate radial velocity of DDO 82, the member of the M 81 group, also should be noted. Its new velocity differs 

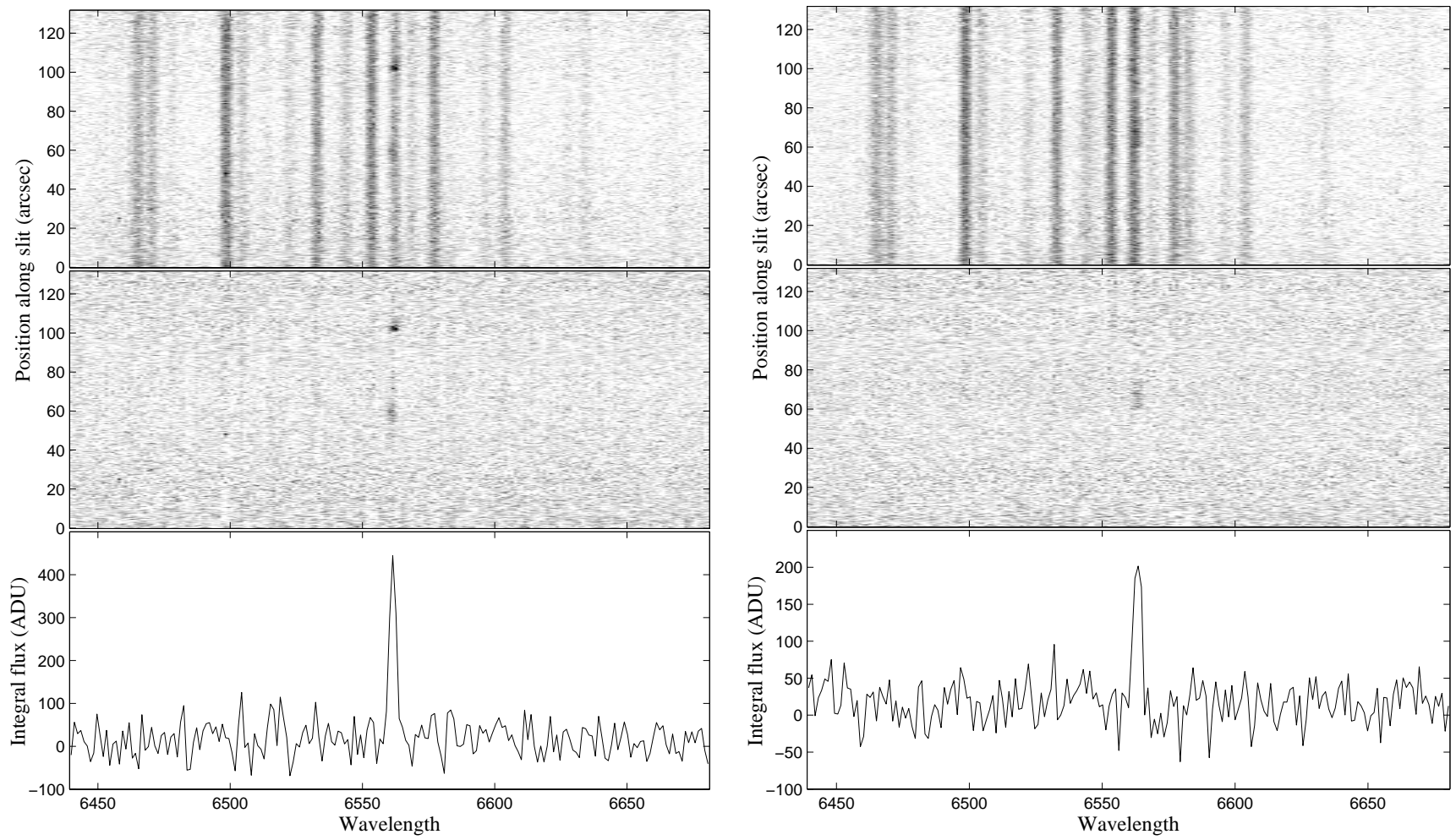

Fig. 3. Reproduction of spectra of two dwarf irregular LSB galaxies in the Maffei/IC 342 group, Cam A and KKH 6, with heliocentric velocities $-49 \mathrm{~km} \mathrm{~s}^{-1}$ and $+23 \mathrm{~km} \mathrm{~s}^{-1}$, respectively. Upper panel: rough spectra; middle panel: after sky subtraction; bottom panel: extracted 1D spectra.
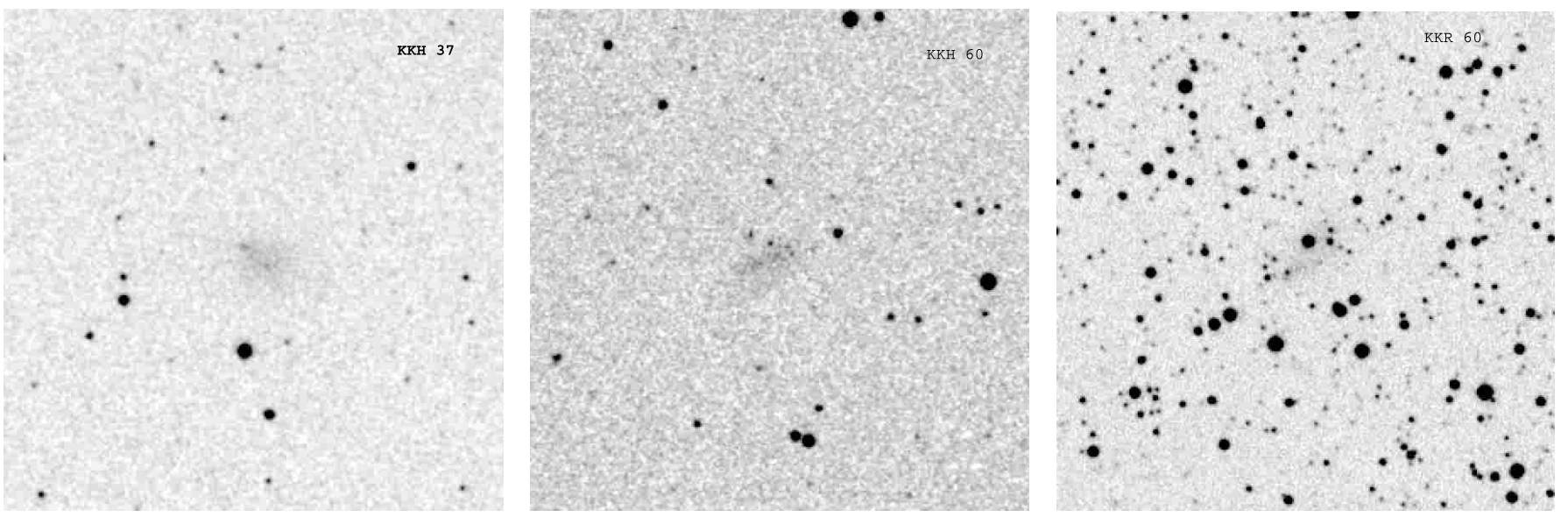

Fig. 4. Red DSS images of three LV galaxy candidates: KKH 37, KKH 60, and KKR 60 with corrected radial velocities, $V_{\mathrm{LG}}$, respectively, $+214,+104$, and $+296 \mathrm{~km} \mathrm{~s}^{-1}$.

from the old velocity in NED by $124 \mathrm{~km} \mathrm{~s}^{-1}$, decreasing the group velocity dispersion.

New radial velocities of three dwarf members of the NGC 6946 group, KKR 55, KKR 56, and KKR 59, make the velocity dispersion in the group lower $\left(67 \mathrm{~km} \mathrm{~s}^{-1}\right.$ instead of $95 \mathrm{~km} \mathrm{~s}^{-1}$ ), which decreases the virial mass-toluminosity ratio of the group from 56 (Karachentsev et al. 2000) to $28 M_{\odot} / L_{\odot}$.

The three objects KKH 37, KKH 60, and KKR 60 are unclear. Their DSS images are shown in Fig. 4. These objects have radial velocities with respect to the Local Group centroid in the range of $[+100,+300] \mathrm{km} \mathrm{s}^{-1}$, and are probable new
Local Volume members. However, their possible nearby location should be confirmed with large-scale images.

Acknowledgements. Support for this work was provided by RFBR grant 01-02-16001 and DFG-RFBR grant 02-02-04012. The Digitized Sky Surveys were produced at the Space Telescope Science Institute under U.S. Government grant NAG W-2166. The images of these surveys are based on photographic data obtained using the Oschin Schmidt Telescope on the Palomar Mountain and the UK Schmidt Telescope. The plates were processed into the present compressed digital form with permission of these institutions. This work made use of the NASA/IPAC Extragalactic Database (NED), 
which is operated by the Jet Propulsion Laboratory, Caltech, under contract with the National Aeronautics and Space Administration.

\section{References}

Barton, W. B., Braun, R., Walterbos, R. A., \& Hoopes, C. G. 1999, AJ, 117, 194

Falco, E. E., Kurtz, M. J., Geller, M. J., et al. 1999, PASP, 111, 438

Hopp, U., \& Schulte-Ladbeck, R. 1991, A\&A, 248, 1

Huchtmeier, W. K., Karachentsev, I. D., \& Karachentseva, V. E. 1997, A\&A, 322, 375

Huchtmeier, W. K., Karachentsev, I. D., Karachentseva, V. E., \& Ehle, M. 2000a, A\&AS, 141, 469

Huchtmeier, W. K., Karachentsev, I. D., \& Karachentseva, V. E. 2000b, A\&AS, 147, 187
Huchtmeier, W. K., Karachentsev, I. D., \& Karachentseva, V. E. 2003, A\&A, 401, 483

Karachentsev, I. D., Sharina, M. E., \& Huchtmeier, W. K. 2000, A\&A, 362,544

Karachentsev, I. D., Karachentseva, V. E., \& Huchtmeier, W. K. 2001, A\&A, 366, $428(\mathrm{KKH})$

Karachentseva, V. E., \& Karachentsev, I. D. 1998, A\&AS, 127, 409 (KK)

Karachentseva, V. E., Karachentsev, I. D., \& Richter, G. M. 1999, A\&AS, 135, 221 (KKR)

Kraan-Korteweg, R., \& Tammann, G. A. 1979, Astron. Nachr., 300, 181

Tully, R. B., Somerville, R. S., Trentham, N., \& Verheijen, M. A. 2002, ApJ, 569, 573 